\title{
Computational Modeling of Polyurea-Coated Composites Subjected to Blast Loads
}

\author{
Tong Hui and Caglar Oskay* \\ Department of Civil and Environmental Engineering \\ Vanderbilt University \\ Nashville, TN 37235
}

\begin{abstract}
This manuscript presents computational modeling and simulation of woven E-glass fiber-reinforced vinyl-ester (EVE) composites and polyurea-coated EVE composites subjected to blast loading. The response of polyurea is idealized based on a temperature and pressure-dependent viscoelastic constitutive model. The response of the EVE layers is modeled based on a multiscale computational damage model that includes adiabatic heating and rate-dependence in the constituent (i.e. matrix and fiber) behavior. Experimentally validated numerical simulations of EVE composite and polyurea-coated EVE composite specimens subjected to blast loading indicate that the proposed models are capable of accurately capturing the inelastic and failure characteristics of the specimens. The significant shock mitigation effect of polyurea coating is demonstrated numerically. Predictive simulations suggest better blast mitigation characteristics with increasing polyurea thickness and confining the perimeter of the polyurea layers.
\end{abstract}

KEYWORDS: Glass fibers; Polymer-matrix composites (PMCs); Impact behavior; Multiscale modeling; Computational Mechanics

\section{INTRODUCTION}

Polyurea coatings have been shown to improve the survivability of structures subjected to blast and impact loading. The blast and impact mitigation characteristics, when combined with cost-effective spray-coating technology for easy application, make polyurea attractive for civilian infrastructure and military protection systems. The mitigative effects of polyurea on a number of different structural materials, including steel $[1,2,3,4,5]$ and composites $[6,7]$ have been subject to a number of recent

\footnotetext{
${ }^{*}$ Corresponding author address: VU Station B\#351831, 2301 Vanderbilt Place, Nashville, TN 37235. Email:
} caglar.oskay@vanderbilt.edu 
investigations. Polyurea exhibits nearly incompressible, viscoelastic response with sensitivity to pressure, strain-rate, and temperature [8]. At high strain-rate, polyurea transitions from rubbery to glassy state, causing an increase in energy absorption capacity [9]. A number of recent experimental and theoretical investigations shed light into the mechanical response characteristics of polyurea such as rate effects, hysteresis and cyclic softening [9], influence of stoichiometry, inertial effects [10, 11], wave propagation characteristics [12], among others. Constitutive laws have also been proposed to model the high-strain rate behavior of polyurea. Amirkhizi et al. [8] developed a viscoelastic constitutive model based on split Hopkinson bar experiments. This model incorporates the effect of temperature based on time-temperature transformation idea, and pressure sensitivity. Li and Lua [13] extended Amirkhizi's model to include nonlinear viscous effects. Elsayed [14] developed a multi-network model, which has the capability to capture hysteresis, rate-effects, plastic deformation, micro-inertia effects and temperature dependence.

Fracture mitigation of polyurea coatings on composite substrates has attracted attention as well. Tekalur et al. [15] conducted an experimental investigation to characterize the effect of polyurea coatings on E-glass reinforced vinyl-ester (EVE) composites subjected to blast. Under blast loading conditions, complex failure mechanisms are observed in EVE composite including permanent deformation, fiber breakage and delamination $[16,17]$, with the extent of damage increasing as a function of blast pressure amplitude. While their results indicate that polyurea coatings are effective in increasing the resistance of composite structures, the mechanisms that lead to such an advantageous behavior are not yet clear.

In this manuscript, we computationally investigate the blast response of glass fiber reinforced composite structures coated with polyurea layers, based on the experimental findings provided by Tekalur et al. [15]. A multiscale computational model for the EVE composite with rate-dependent damage evolution and adiabatic heating effects is devised. Blast response of EVE composite is simulated using the proposed model and numerical results are compared to the experimental data. The analysis of the blast response of polyurea-coated EVE composites with three different layup configurations is conducted by employing the polyurea constitutive model proposed by Amirkhizi et al. [8]. A predictive parametric investigation is conducted to characterize the effects of the thickness as well as the confinement of the polyurea layer on blast mitigation capability. The numerical analysis 
reveals better survivability characteristics with increasing thickness and through confinement of the polyurea layer.

The remainder of this manuscript is organized as follows: In Section 2, a strain rate- and temperature dependent multiscale constitutive model for EVE composite is presented along with the calibrated properties for quasi-static and high rate loading. In Section 3, the temperature- and pressure-dependent polyurea model is described. Section 4 presents the numerical simulations of EVE and polyurea-coated EVE composite panels subjected to blast loading. Predictive investigations of the effect of polyurea thickness and confinement are discussed. Section 5 provides the conclusions drawn from the numerical investigations.

\section{MODELING APPROACH}

We consider a composite structure occupying domain $\Omega$, subjected to high-rate loading. The dynamic response of the composite is governed by the momentum balance equation:

$$
\boldsymbol{\nabla} \cdot \overline{\boldsymbol{\sigma}}(\mathbf{x}, t)+\overline{\mathbf{b}}(\mathbf{x}, t)=\bar{\rho}(\mathbf{x}, t) \ddot{\overline{\mathbf{u}}}(\mathbf{x}, t) ; \quad \mathbf{x} \in \Omega ; \quad t \in\left[0, t_{\mathrm{o}}\right]
$$

in which, $\overline{\boldsymbol{\sigma}}, \overline{\mathbf{u}}$ denote the stress and displacement fields, respectively; $\overline{\mathbf{b}}$ the body force; $\bar{\rho}$ the density; and, $\mathbf{x}$ and $t$ denote spatial and temporal coordinates, respectively; Superscribed dot denotes material time derivative. Overbar indicates that the corresponding field is associated with an equivalent homogeneous representation of the heterogeneous medium. The structure is subjected to initial and boundary conditions:

$$
\begin{aligned}
\overline{\mathbf{u}}(\mathbf{x}, t) & =\hat{\mathbf{u}}(\mathbf{x}) ; \quad \mathbf{x} \in \Omega ; \quad t=0 \\
\dot{\mathbf{u}}(\mathbf{x}, t) & =\hat{\mathbf{v}}(\mathbf{x}) ; \quad \mathbf{x} \in \Omega ; \quad t=0 \\
\overline{\mathbf{u}}(\mathbf{x}, t) & =\tilde{\mathbf{u}}(\mathbf{x}, t) ; \quad \mathbf{x} \in \Gamma_{u} ; \quad t \in\left[0, t_{\mathrm{o}}\right] \\
\overline{\boldsymbol{\sigma}}(\mathbf{x}, t) \cdot \mathbf{n} & =\tilde{\mathbf{t}}(\mathbf{x}, t) ; \quad \mathbf{x} \in \Gamma_{t} ; \quad t \in\left[0, t_{\mathrm{o}}\right]
\end{aligned}
$$

where, $\hat{\mathbf{u}}$ and $\hat{\mathbf{v}}$ are prescribed initial displacement and velocity fields, respectively; $\tilde{\mathbf{u}}$ and $\tilde{\mathbf{t}}$ the prescribed displacements and tractions on the boundaries $\Gamma_{u}$ and $\Gamma_{t}$, respectively, $\left(\Gamma=\Gamma_{u} \cup \Gamma_{t}\right.$ and $\left.\Gamma_{u} \cap \Gamma_{t}=\emptyset\right)$; and; $\mathbf{n}$ the unit normal to $\Gamma_{t}$. 
In this manuscript, the failure response of the composite structure is evaluated by employing explicit finite element method to evaluate Eqs. 1-5, and appropriate constitutive models for constituent materials with prescribed blast loading profiles (i.e., Eq. 5). In the current study, we focus our attention to relatively low blast amplitudes, in which through thickness Hugoniot effects, as well as wave reflections due to microstructural heterogeneity play a negligible role in the overall response and failure. When the applied loading rate and the loading amplitude is high, the effects of shock waves, wave dispersion and microinertia due to heterogeneous microstructure of the composite become significant, which may not be accurately captured by explicit finite element modeling with Eq. 1 . Shock waves are propagating surfaces of discontinuities in mass density, stress, velocity as well as other state variables of the material. Shock wave propagation in composite materials has been previously investigated based on the hydrodynamic theory [18], and equations of state describing the shock response have been reported in the literature (see e.g., Ref. [19]). The hydrodynamic theory based investigations is sufficiently accurate in describing the shock response of composites at high loading amplitudes (i.e., when the pressure magnitudes are in the order of the elastic moduli). At high rates of loading, deformation wave interaction mechanisms (i.e., reflections and refractions) at the microscopic scales become dominant and the dispersion effects due to heterogeneous microstructure must be taken into account. The realization of this phenomena dates back to the classical works of Cosserat and Cosserat [20], Mindlin [21], and Eringen [22]. The effects of micro-inertia and dispersion have been recently addressed through a number of approaches such as gradient enhancement [23], by mathematical homogenization with multiple spatial and temporal scales [24], time-harmonic Bloch expansions [25], scale bridging based on Hamilton's principle [26], and models based on Mindlin's theory [27].

\section{CONSTITUTIVE FAILURE MODEL FOR COMPOSITE CONSTITUENTS UNDER HIGH-RATE LOADING}

The failure response of the composite material is modeled based on the eigendeformation-based reduced order computational homogenization (EHM) method recently proposed by Oskay and co-workers $[28,29,30]$. In the EHM method, the microstructural response at the scale of the representative volume element (RVE) is numerically evaluated based on the idea of precomputing 
certain microstructural information (e.g., localization operators, concentration tensors, influence functions) by evaluating linear elastic RVE scale simulations, prior to the analysis of the macroscale structure. The inelastic response fields within the RVE are taken to be piecewise constant (in space) within subdomains of the RVE corresponding to each phase. The details of the EHM method are presented elsewhere [28] and skipped in this discussion. In this section, we propose a strain-rate dependent and temperature-dependent constitutive formulation to describe the failure response of the composite constituents. Strain-rate dependence is introduced through a Perzyna-type visco-damage formulation to account for embrittlement and hardening observed at high rates, as well as to avoid mesh sensitivity observed in standard continuum damage formulations. Temperature-dependence is introduced to investigate the effect of localized adiabatic heating on the blast response, which has been previously argued to have a significant effect on localization of damage under high rate loading. [31]

The composite material is considered to be formed by the repetition of a locally periodic microstructure, which consists of $n_{\text {ph }}$ phases (e.g., matrix and fiber ). The constitutive behavior of each composite constituent is idealized based on a rate- and temperature-dependent continuous damage mechanics model. The phase-average stress within phase, $\eta$, is expressed as:

$$
\boldsymbol{\sigma}^{(\eta)}=\mathbf{L}^{(\eta)}: \mathbf{e}^{(\eta)}=\mathbf{L}^{(\eta)}:\left(\epsilon^{(\eta)}-\boldsymbol{\mu}^{(\eta)}\right)
$$

where, $\boldsymbol{\epsilon}^{(\eta)}, \boldsymbol{\mu}^{(\eta)}$ and $\mathbf{e}^{(\eta)}$ are phase average total, inelastic and elastic strain tensors, respectively; $\mathbf{L}^{(\eta)}$ the tensor of elastic moduli; and, colon denotes double inner product operator.

Under high-rate loading conditions, the phase-average inelastic strain is expressed in terms of the scalar damage variable, $\omega^{(\eta)} \in[0,1)$, indicating the state of damage, and localized adiabatic heating induced thermal strains:

$$
\sum_{\gamma}\left[\delta_{\eta \gamma} \mathbf{I}-\mathbf{P}^{(\eta \gamma)} \omega^{(\eta)}\right]: \boldsymbol{\mu}^{(\gamma)}=\omega^{(\eta)} \mathbf{A}^{(\eta)}: \bar{\epsilon}(\mathbf{x}, t)-\alpha^{(\eta)}\left[1-\omega^{(\eta)}\right]\left[T^{(\eta)}-T_{\text {ref }}\right] \boldsymbol{\delta}
$$

in which, $\bar{\epsilon}$ is the RVE average (macroscale) strain; $\alpha^{(\eta)}$ the coefficient of thermal expansion; $T^{(\eta)}$ and $T_{\text {ref }}$ the current and reference temperatures, respectively; $\boldsymbol{\delta}$ and $\mathbf{I}$ denote the second and fourth order identity tensors, respectively; and, $\delta_{\eta \gamma}=1$ if $\eta=\gamma$ and vanish otherwise. the fourth order coefficient tensors, $\mathbf{P}^{(\eta \gamma)}$ and $\mathbf{A}^{(\eta)}$ are expressed as: 


$$
\mathbf{P}^{(\eta \gamma)}=\frac{1}{\left|\Theta^{(\eta)}\right|} \int_{\Theta^{(\eta)}} \int_{\Theta^{(\gamma)}} \mathbf{g}(\mathbf{y}, \hat{\mathbf{y}}) d \hat{\mathbf{y}} d \mathbf{y} ; \quad \mathbf{A}^{(\eta)}=\frac{1}{\left|\Theta^{(\eta)}\right|} \int_{\Theta^{(\eta)}} \mathbf{I}+\mathbf{G}(\mathbf{y}) d \mathbf{y}
$$

where, $\Theta^{(\eta)}$ denotes the domain of the constituent, $\eta$; and, $\mathbf{g}$ and $\mathbf{G}$ are the inelastic deformation-induced and elastic polarization functions of the RVE problem, which are numerically evaluated based on the EHM method. Equation 7 ensures that the equilibrium at the microscopic (i.e. RVE) scale is satisfied.

The RVE average (macroscale) stress of the overall composite material is expressed in terms of the macroscale strain and the phase average inelastic strains as:

$$
\overline{\boldsymbol{\sigma}}=\overline{\mathbf{L}}: \overline{\boldsymbol{\epsilon}}+\sum_{\gamma} \overline{\mathbf{M}}^{(\gamma)}: \boldsymbol{\mu}^{(\gamma)}
$$

in terms of the coefficient tensors, $\overline{\mathbf{M}}^{(\gamma)}$ and $\overline{\mathbf{L}}$,

$$
\overline{\mathbf{M}}^{(\gamma)}=\sum_{\eta} \mathbf{L}^{(\eta)}:\left[c^{(\eta)} \mathbf{P}^{(\eta \gamma)}-\delta_{\eta \gamma} \mathbf{I}\right] ; \quad \overline{\mathbf{L}}=\sum_{\eta} c^{(\eta)} \mathbf{L}^{(\eta)}: \mathbf{A}^{(\eta)}
$$

where, $c^{(\eta)}$ denotes the volume fraction of phase $\eta$ within the RVE.

Localized adiabatic heating during high-rate loading is taken to be due to reversible elastic deformations, as well as the irreversible damage process [32, 33]:

$$
\rho^{(\eta)} C_{p}^{(\eta)} \dot{T}^{(\eta)}=\Upsilon^{(\eta)} \sigma^{(\eta)}: \dot{\boldsymbol{\mu}}^{(\eta)}-3 K^{(\eta)} \alpha^{(\eta)}\left(1-\omega^{(\eta)}\right) \operatorname{tr}\left(\dot{\mathbf{e}}^{(\eta)}\right) T^{(\eta)}+\frac{1}{3} \operatorname{tr}\left(\boldsymbol{\sigma}^{(\eta)}\right) \operatorname{tr}\left(\dot{\mathbf{e}}^{(\eta)}\right)
$$

where, $C_{p}^{(\eta)}$ and $\rho^{(\eta)}$ represent the specific heat at constant pressure and density of phase $\eta$, respectively; $\Upsilon^{(\eta)}$ the ratio of inelastic work converted to heat; and, $K^{(\eta)}$ denotes bulk modulus; and, $\operatorname{tr}(\cdot)$ is the trace operator.

We consider a damage evolution model with viscous regularization of the Perzyna type [34] to idealize the rate-dependent response of the phases. Similar rate-dependent damage models with viscoplastic regularization have been proposed for failure modeling of other quasi-brittle materials $[35,36]$. Rate-independent continuum damage mechanics models are well-known to exhibit spurious mesh sensitivity when loading extends to the softening regime. This phenomenon is marked by the localization of strains within the size of a finite element. Viscous regularization of the damage model employed in this study has been shown to alleviate damage localization and spurious mesh sensitivity $[37,38]$. 
In this manuscript, the effect of localized heating on the rate-dependent damage evolution is introduced. Let $f$ be the temperature-dependent damage potential function in the form:

$$
f\left(v^{(\eta)}, r^{(\eta)}, T^{(\eta)}\right)=\phi\left(v^{(\eta)}\right)-\phi\left(r^{(\eta)}\right)\left[1-\left(\frac{T^{(\eta)}-T_{\mathrm{ref}}^{(\eta)}}{T_{\mathrm{m}}^{(\eta)}-T_{\mathrm{ref}}^{(\eta)}}\right)\right] \leq 0
$$

in which, $v^{(\eta)}$ is the phase damage equivalent strain; $r^{(\eta)}$ the damage hardening variable; and, $T_{\mathrm{m}}^{(\eta)}$ the melting temperature of constituent phase $\eta . \phi$ is the damage evolution function. The evolution equations for the damage and hardening variables are given as:

$$
\dot{\omega}^{(\eta)}=\lambda \frac{\partial \phi}{\partial v^{(\eta)}} ; \quad \dot{r}^{(\eta)}=\lambda
$$

in which, the consistency parameter, $\lambda$, is described by the power law expression:

$$
\lambda=\frac{1}{q^{(\eta)}}\langle f\rangle^{p^{(\eta)}}
$$

where, $\langle\cdot\rangle \equiv((\cdot)+|\cdot|) / 2$ denotes MacCauley brackets; and $p^{(\eta)}$ and $q^{(\eta)}$ are material parameters describing the rate-dependent response.

The phase damage equivalent strain is defined as

$$
v^{(\eta)}=\sqrt{\frac{1}{2}\left(\mathbf{F}^{(\eta)} \hat{\boldsymbol{\epsilon}}^{(\eta)}\right): \mathbf{L}^{(\eta)}:\left(\mathbf{F}^{(\eta)} \hat{\boldsymbol{\epsilon}}^{(\eta)}\right)}
$$

in which, $\hat{\boldsymbol{\epsilon}}^{(\eta)}$ is the principal strain tensor; $\mathbf{F}^{(\eta)}=\operatorname{diag}\left(h_{1}^{(\eta)}, h_{2}^{(\eta)}, h_{3}^{(\eta)}\right)$ is a diagonal weighting matrix introduced to differentiate damage growth rate in tensile and compressive loading:

$$
h_{\xi}^{(\eta)}=\frac{1}{2}+\frac{1}{\pi} \arctan \left(c_{1}^{(\eta)} \hat{\varepsilon}_{\xi}^{(\eta)}\right) ; \quad \xi=1,2,3
$$

where, $\hat{\varepsilon}_{\xi}^{(\eta)}$ are components of $\hat{\boldsymbol{\epsilon}}^{(\eta)}$; and, $c_{1}^{(\eta)}$ is a material parameter.

A power law is considered to characterize the damage evolution function, $\phi$, which increases monotonically as a function of the damage equivalent strain to satisfy Clausius-Duhem inequality for thermodynamic consistency:

$$
\phi\left(v^{(\eta)}\right)=a^{(\eta)}\left\langle v^{(\eta)}\right\rangle^{b^{(\eta)}} ; \phi\left(v^{(\eta)}\right) \leq 1
$$

in which, $a^{(\eta)}$ and $b^{(\eta)}$ are material parameters. 


\section{POLYUREA MODEL}

The mechanical response of polyurea subjected to blast loading is modeled using the temperature- and pressure-dependent viscoelastic constitutive law proposed by Amirkhizi et al. [8]. Cauchy stress tensor is expressed in terms of the hereditary integral as a function of the shear relaxation modulus and the bulk modulus as:

$$
\overline{\boldsymbol{\sigma}}=\int_{0}^{t} 2 G^{(p)}(t, \tau) \mathbf{D}^{\prime}(\tau) d \tau+K^{(p)} \frac{\ln J}{J} \boldsymbol{\delta}
$$

where, $\mathbf{D}^{\prime}(t)$ is the deviatoric component of the deformation-rate tensor, $\mathbf{D}=\left(\mathbf{L}^{T}+\mathbf{L}\right) / 2$;

$\left(\mathbf{L}=\dot{\mathbf{F F}}^{-1}\right)$ is the velocity gradient and $\mathbf{F}$ the deformation gradient $)$; and, $J=\operatorname{det}(\mathbf{F})$ the jacobian of the deformation gradient. The bulk modulus of polyurea, $K^{(p)}$ is taken to be a linear function of the temperature:

$$
K^{(p)}(T)=K^{(p)}\left(T_{\text {ref }}\right)+m^{(p)}\left(T-T_{\text {ref }}\right)
$$

in which, $K^{(p)}\left(T_{\text {ref }}\right)$ is the bulk modulus of polyurea at reference temperature; and, $m^{(p)}$ is the temperature sensitivity parameter.

The shear relaxation modulus, $G^{(p)}$, is expressed based on an exponential (Prony) series representation:

$$
G^{(p)}(t, \tau)=\frac{T(\tau)}{T_{\text {ref }}} G_{\infty}^{(p)}\left(1+\sum_{i=1}^{n} p_{i}^{(p)} \exp \left[\frac{-(\xi(t)-\xi(\tau))}{q_{i}^{(p)}}\right]\right) ; \quad \tau \in[0, t]
$$

where, $G_{\infty}$ is the steady state shear modulus of polyurea; $p_{i}^{(p)}$ and $q_{i}^{(p)}$ are material constants; and:

$$
\xi(t)=\int_{0}^{t} \frac{1}{a(T(\tau), P(\tau))} d \tau
$$

in which, $P=-\operatorname{tr}(\boldsymbol{\sigma}) / 3$ is pressure; $a(T, P)$ is the Williams-Landel-Ferry (WLF) empirical time-temperature-pressure shift function of the form:

$$
\log (a(T, P))=\frac{A^{(p)}\left(T-C_{t p}^{(p)} P-T_{\mathrm{ref}}\right)}{B^{(p)}+T-C_{t p}^{(p)} P-T_{\mathrm{ref}}}
$$

where, $A^{(p)}$ and $B^{(p)}$ are time-temperature coefficients; and, $C_{t p}^{(p)}$ the time-pressure coefficient.

Viscous dissipation causes localized adiabatic heating in polyurea during blast loading:

$$
\dot{T}=\frac{1}{C_{\mathrm{v}}^{(p)}} \dot{W}_{d}=\frac{2 G_{\infty}^{(p)}}{C_{\mathrm{v}}^{(p)}} \frac{T(t)}{T_{\mathrm{ref}}} \sum_{i=1}^{n} \frac{p_{i}^{(p)}}{q_{i}^{(p)}} \epsilon_{d}^{i}(t): \epsilon_{d}^{i}(t)
$$

in which, $W_{d}$ is the dissipated work per unit reference volume; and, $C_{\mathrm{v}}^{(p)}$ is the heat capacity per 
original volume; and,

$$
\epsilon_{d}^{i}(t)=\int_{0}^{t} \exp \left[\frac{-(\xi(t)-\xi(\tau))}{q_{i}^{(p)}}\right] \mathbf{D}^{\prime}(\tau) d \tau
$$

The material parameters of polyurea are calibrated based on Kolsky (Split Hopkinson) pressure bar experiments. The polyurea material parameters employed in our simulations are provided by Ref. [8] and summarized in Table 1.

\section{BLAST SIMULATION OF EVE/POLYUREA COMPOSITES}

In this section, we numerically investigate the response of EVE and polyurea-coated EVE composite specimens subjected to blast loading. We employ specimen geometry and loading conditions of the experimental investigations provided by Tekalur and co-workers [15]. The geometry and discretization of composite panels are illustrated in Fig. 1. The EVE panels are $230 \mathrm{~mm} \times 102 \mathrm{~mm} \times 6 \mathrm{~mm}$ in length, width and thickness, respectively. The fill and warp fibers are oriented along $x_{1}$ and $x_{2}$ directions respectively. The panel is simply supported over a span of $152 \mathrm{~mm}$ along the vertical direction. The blast loading is applied at the center of the panel within a circular area with radius of $38 \mathrm{~mm}$. Only a quadrant of the panel is modeled and simulated by exploiting the symmetry of the panel geometry, loading and boundary conditions. We consider four panel configurations: (1) EVE panel only (E-configuration; Fig. 1b); (2) $6 \mathrm{~mm}$ polyurea coating at the back face (E $\mid$ P-configuration; Fig. 1d); (3) 6mm polyurea coating at the front face $(\mathrm{P} \mid$ E-configuration;

Fig. 1e); (4) 6mm polyurea layer sandwiched by two $3 \mathrm{~mm}$-thick EVE panels (E|P|E-configuration; Fig. 1f). Fig. 2 illustrates the blast loading profiles employed in this study. The shock wave profile is characterized by a peak blast (reflected wave) amplitude, $P_{\text {ref }}$, incident wave amplitude, $P_{\text {inc }}$, and the time duration, $\lambda=1.5 \mathrm{~ms}$, within which reflected wave amplitude decays to incident wave amplitude. Linear and nonlinear profiles are considered. The nonlinear wave profile is generated by the expression:

$$
P=\frac{\lambda P_{\text {inc }} P_{\text {ref }}}{P_{\text {inc }} \lambda+\left(P_{\text {ref }}-P_{\text {inc }}\right) t}
$$




\section{Calibration of E-Glass Vinyl Ester (EVE) Constituent Properties}

The proposed multiscale computational model is applied to idealize the response of EVE composite employed in the experimental study provided in Ref. [15]. The elastic and failure properties are calibrated using available experimental data on quasi-static and dynamic response of EVE composite specimens provided in references $[31,39]$. The representative volume element (RVE) is idealized as a composition of three constituents: the vinyl ester matrix, fiber tows along the fill- and warp directions. The RVE of the woven composite material employed in the simulations is shown in Fig. 3. The microstructure of the composite contains $60.5 \%$ fiber by volume, with an unbalanced construction having $59 \%$ and $41 \%$ of fibers oriented along the warp and fill directions, respectively. The size of the RVE is $10 \mathrm{~mm} \times 12.5 \mathrm{~mm} \times 0.625 \mathrm{~mm}$ along the fill, warp and thickness directions, respectively. Density, specific heat, and coefficient of thermal expansion for matrix and fiber phases employed in our investigations are obtained from Ref. [40]. The experimentally determined quasi-static elastic properties of matrix and fiber are [41,31]: $E_{0}^{(m)}=3.4 \mathrm{GPa}, E^{(f)}=70 \mathrm{GPa}$, and $\boldsymbol{v}^{(m)}=v^{(f)}=0.15$. At high strain rates the elastic modulus of overall composite is higher than those at quasi-static conditions [39, 42]. At blast loading rates and temperature ranges considered in this study, E-glass fibers do not display significant strain rate effects. The elastic modulus of vinyl-ester matrix is taken to be a function of strain-rate based on a power law [39]:

$$
E^{(m)}=E_{0}^{(m)}\left(1+\left(\frac{\dot{\boldsymbol{\varepsilon}}^{(m)}}{\dot{\boldsymbol{\varepsilon}}_{0}^{(m)}}\right)^{N}\right)
$$

where, $\dot{\varepsilon}_{0}^{(m)}=2060 / \mathrm{s}$ is the reference strain rate; and, $N=0.73$. The quasi-static uniaxial compression and tension experiments conducted by Tekalur et al. [31], as well as the dynamic testing by Oguni and Ravichandran [39] are employed to calibrate the failure properties of the constituent phases. For each constituent, parameters $a^{(\gamma)}$ and $b^{(\gamma)}$ define the evolution of damage as a function of loading history; $c_{1}^{(\gamma)}$ and $c_{2}^{(\gamma)}$ model the strength anisotropy along the compression and tensile directions; and, $p^{(\gamma)}$ and $q^{(\gamma)}$ model the rate-dependent behavior. The fiber phase response is taken to be rate-independent [39] within the loading rates and temperature ranges considered in this study. The calibrated material parameters for each phase are summarized in Table 2 and the stress-strain curves at high and low strain rate are plotted in Fig. 4. The experimentally observed strength of the overall composite and the 
calibrated model predictions are compared in Table 3, which are in reasonable agreement.

\section{Blast Response of EVE Panels}

The E-configuration panel is subjected to three loading profiles to assess the predictive capability of the proposed model and investigate the effects of loading rate and damage induced adiabatic heating on the blast response. The loading profiles are parameterized as: $P_{\text {inc }}=0.62 \mathrm{MPa}$ and $P_{\text {ref }}=1.57 \mathrm{MPa}$ for linear and nonlinear models; and, $P_{\mathrm{inc}}=0.45 \mathrm{MPa}$ and $P_{\text {ref }}=1.28 \mathrm{MPa}$ for nonlinear model only. Figure 5a compares the numerical predictions of linear and nonlinear blast loading with incident wave amplitude of $0.62 \mathrm{MPa}$, as well as the experiments. The predicted displacement histories along the thickness direction at the center of the plate (center-point displacement, $u_{c}$ ) are in reasonable agreement with the experimental observations. The discrepancy between the predictions of linear and nonlinear blast loading models illustrates the effect of sensitivity of panel response on the accuracy of the input blast load profiles. The more realistic non-linear blast loading model provides a better fit with the experimental results especially at the later stages of deformation. In the remainder of the simulations, only the nonlinear blast profile is employed. The center-point displacement comparison between numerical simulation and experiment for incident wave amplitude of $0.45 \mathrm{MPa}$ is plotted in Fig. 5b. In contrast to high incident wave amplitude simulation, the specimen does not completely fail and the displacement rebounds back after $1.1 \mathrm{~ms}$.

In all numerical investigations, the maximum observed strain rate throughout the specimen and loading history does not exceed $20 / \mathrm{s}$. The rate effects, which are pronounced in the matrix material at high strain rate ( i.e. $>1000 / \mathrm{s}$ [39]), therefore, do not significantly affect the blast response. In adiabatic heating process, maximum damage-induced temperature rise is achieved by setting $\Upsilon$ equal to 1 signifying that all of the damage work is converted into heat. The thermal profile of the composite specimen computed based on Eq. 11 throughout the loading history indicates that maximum temperature rise due to damage induced adiabatic heating is confined to less than $10 \mathrm{~K}$. The burn observed along the surface of the specimens revealed in experimental studies is therefore unlikely to be due to damage induced heating.

\section{Blast Response of Polyurea-Coated EVE Composites}

We conducted a series of simulations to numerically investigate the mitigative effect of polyurea-coating on the blast survivability of EVE composite panels. Three polyurea-EVE composite 
configurations illustrated in Figs. 1d-f are subjected to blast load profiles. The incident pressure and reflected pressure amplitudes of the nonlinear blast load profiles are: $0.75 \mathrm{MPa}$ and 2.2 $\mathrm{MPa}$, respectively, for $\mathrm{E} \mid \mathrm{P}$ - and $\mathrm{P} \mid \mathrm{E}-$ configurations; and, $0.75 \mathrm{MPa}$ and $2.01 \mathrm{MPa}$, respectively, for $\mathrm{E}|\mathrm{P}| \mathrm{E}$ configuration. Figure 6 illustrates simulated and experimentally observed center-point displacement (normalized by the combined thickness of the polyurea layer and EVE composite panel, $d_{t}$ ) histories for $\mathrm{P}|\mathrm{E}, \mathrm{E}| \mathrm{P}$ and $\mathrm{E}|\mathrm{P}| \mathrm{E}$ configurations, respectively. For all three configurations, the numerical predictions display reasonable agreement with the experimental observations, pointing to the adequacy of the proposed models in capturing the failure response of composite specimens. The $\mathrm{P} \mid \mathrm{E}$ and $\mathrm{E}|\mathrm{P}| \mathrm{E}$ configurations resist the blast loading with only partial damage under the applied incident wave amplitude of $0.75 \mathrm{MPa}$, that is illustrated in Figs. 6a and $6 \mathrm{c}$ as the curve-down of center-point displacements. In contrast, the $\mathrm{E} \mid \mathrm{P}$ configuration fails under the applied blast magnitude. The highest mitigation effect is observed in the $\mathrm{E}|\mathrm{P}| \mathrm{E}$ configuration, in which the center-point displacement completely rebounds. The superior performance of $\mathrm{P} \mid \mathrm{E}$ configuration (compared to the $\mathrm{E} \mid \mathrm{P}$ configuration) is attributed to its higher resistance against compressive and shear failure of the substrate, which are predominant failure mechanisms [15], as well as dissipation of blast pressure within polyurea layer prior to reaching the composite substrate. All three simulations indicate that the presence of polyurea coating significantly mitigates blast response as evidenced by reductions in the center-point displacement magnitudes compared to the E-configuration despite higher applied blast pressure magnitudes for polyurea-coated configurations. Figure 7 illustrates snapshots of simulated matrix damage and deformation profiles during the blast loading of $\mathrm{E}$ and $\mathrm{E} \mid \mathrm{P}$ configurations compared to experimental observations [15]. In all simulations, the maximum rise in temperature within polyurea layers is $2 \mathrm{~K}$. The highest strain-rates within the polyurea layers throughout the loading history are 150/s, 800/s and 50/s for the $\mathrm{P}|\mathrm{E}, \mathrm{E}| \mathrm{P}$ and $\mathrm{E}|\mathrm{P}| \mathrm{E}$ configurations, respectively.

\section{Effect of polyurea confinement}

The polyurea is a nearly incompressible material. We investigated the effect of confinement of the polyurea layers on the blast response and mitigation characteristics. The confinement effect is presented only for the $\mathrm{E}|\mathrm{P}| \mathrm{E}$ configuration, since the confinement effect is independent of the composite layup. The confinement effect is achieved by constraining the boundary displacement of the polyurea layer along $x_{1}$ and $x_{2}$ directions throughout the perimeter of the specimen. Figure 8 
compares the center-point displacements of the confined and unconfined $\mathrm{E}|\mathrm{P}| \mathrm{E}$ configurations. The center-point displacements at the initial stages of the loading are similar. In contrast, the peak displacement for the confined configuration is approximately $30 \%$ smaller compared to the unconfined configuration. The deformation for the unconfined configuration is significantly smaller throughout the rest of the loading history.

Effect of polyurea coating thickness

A parametric investigation is conducted to identify the effect of the thickness of the polyurea coatings on the blast mitigation effectiveness. $\mathrm{P}|\mathrm{E}, \mathrm{E}| \mathrm{P}$ and $\mathrm{E}|\mathrm{P}| \mathrm{E}$ panels with $3 \mathrm{~mm}-, 6 \mathrm{~mm}$ - and $12 \mathrm{~mm}$-thick polyurea layers are subjected to blast loading. Figure 9 shows the center-point displacement histories for $\mathrm{P}|\mathrm{E}, \mathrm{E}| \mathrm{P}$ and $\mathrm{E}|\mathrm{P}| \mathrm{E}$ configurations, respectively. For all three configurations, the increasing thickness of polyurea monotonically reduces the blast induced damage and damage induced deformations in the EVE composite layers. In the case of $\mathrm{P} \mid \mathrm{E}$ configuration, a $3 \mathrm{~mm}$-thick polyurea layer fails to mitigate the blast response, and complete failure of the EVE composite layer is observed. In the case of $\mathrm{E} \mid \mathrm{P}$ configuration, the $12 \mathrm{~mm}$-thick polyurea layer mitigates complete failure of the EVE composite layer, in contrast with the $3 \mathrm{~mm}$ - and $6 \mathrm{~mm}$-thick polyurea layer configurations. All $\mathrm{E}|\mathrm{P}| \mathrm{E}$ panels with $3 \mathrm{~mm}-, 6 \mathrm{~mm}$ - and $12 \mathrm{~mm}$-thick polyurea layers survive the blast loading, with no significant damage accumulation within the EVE composite layer in the case of $12 \mathrm{~mm}$-thick polyurea configuration.

\section{CONCLUSIONS}

This manuscript investigated the blast mitigation effect of polyurea-coating on woven E-glass fiber-reinforced vinyl-ester (EVE) composites. A new multiscale computational failure model for EVE composites that accounts for strain-rate effects as well as damage induced localized adiabatic heating is proposed to accurately model the response of EVE composites subjected to blast loading. The predictions of the blast response of EVE composite specimens, as well as the polyurea-coated EVE composite specimens using the proposed computational approach display a very reasonable agreement with the experimental observations.

The following observations are made regarding the blast response of polyurea-coated EVE composite systems: (1) Strain rate hardening, and temperature have limited influence on deformation 
response and damage accumulation characteristics of EVE composite panels subjected to blast loading at the amplitudes investigated in this study. (2) Among the specimen configurations, the numerical simulations confirm that sandwich configurations have significantly better mitigation characteristics compared to coating the EVE composite layers with polyurea. (3) Thickness of the polyurea has a significant affect in blast mitigation effectiveness with thicker coatings leading to better protection. (4) Peripheral confinement of the polyurea layer increases the blast mitigation effectiveness, which points to the observation that stiffer polyurea with similar visco-elastic response characteristics has a potential to display better blast mitigation characteristics.

\section{ACKNOWLEDGMENTS}

The financial support of the National Science Foundation, CMMI Hazard Mitigation and Structural Engineering program (Grant \#:0856168) is gratefully acknowledged.

\section{REFERENCES}

[1] Amini, M., Isaacs, J. and Nemat-Nasser, S. (2010). Investigation of Effect of Polyurea on Response of Steel Plates to Impulsive Loads in Direct Pressure-Pulse Experiments, Mech. Mater., 42(6): 628-639.

[2] Amini, M., Simon, J. and Nemat-Nasser, S. (2010). Numerical Modeling of Effect of Polyurea on Response of Steel Plates to Impulsive Loads in Direct Pressure-pulse Experiments, Mech. Mater., 42(6): 615-627.

[3] Grujicic, M., Pandurangan, B. and He, T. (2010). Computational Investigation of Impact Energy Absorption Capability of Polyurea Coatings via Deformation-Induced Glass Transition, Mater. Sci. Eng., A, 527: 7741-7751.

[4] Xue, L., Mock, W. and Belytschko, T. (2010). Penetration of Dh-36 Steel Plates with and without Polyurea Coating, Mech. Mater., 42: 981-1003.

[5] El Sayed, T., Mock, W., Mota, A., Fraternali, F. and Ortiz, M. (2009). Computational Assessment of Ballistic Impact on A High Strength Structural Steel/Polyurea Composite Plate, Comput. Mech., 43(4): 525-534. 
[6] Grujicic, M., Bell, W., Pandurangan, B. and He, T. (2010). Blast-Wave Impact-Mitigation Capability of Polyurea When Used as Helmet Suspension Pad Material, Mater. Des., 31: 4050-4065.

[7] Bahei-El-Din, Y., Dvorak, G. and Fredricksen, O. (2006). A Blast-Tolerant Sandwich Plate Design with A Polyurea Interlayer, Int. J. Solids Struct., 43(25-26): 7644-7658.

[8] Amirkhizi, A., Isaacs, J., McGee, J. and Nemat-Nasser, S. (2006). An Experimentally-Based Viscoelastic Constitutive Model for Polyurea, Including Pressure and Temperature Effects, Philos. Mag., 86(36): 5847-5866.

[9] Yi, J., Boyce, M., Lee, G. and Balizer, E. (2006). Large Deformation Rate-Dependent Stress-Strain Behavior of Polyurea and Polyurethanes, Polym. J., 47(1): 319-329.

[10] Fragiadakis, D., Gamache, R., Bogoslovov, R. and Roland, C. (2010). Segmental Dynamics of Polyurea: Effect of Stoichiometry, Polym. J., 51(1): 178-184.

[11] Roland, C., Twigg, J., Vu, Y. and Mott, P. (2007). High Strain Rate Mechanical Behavior of Polyurea, Polym. J., 48(2): 574-578.

[12] Ravi-Chandar, K. and Niemczura, J. (2011). Tensile Shock Waves in Rubber, in Bulletin of the American Physical Society, volume 56, American Physical Society.

[13] Li, C. and Lua, J. (2009). A Hyper-Viscoelastic Constitutive Model for Polyurea, Mater. Lett., 63(11): 877-880.

[14] El Sayed, T., Mota, A., Fraternali, F. and Ortiz, M. (2008). A Variational Constitutive Model for Soft Biological Tissues, J. Biomech., 41(7): 1458-1466.

[15] Tekalur, S., Shukla, A. and Shivakumar, K. (2008). Blast Resistance of Polyurea Based Layered Composite Materials, Compos. Struct., 84(3): 271-281.

[16] Librescu, L. and Nosier, A. (1990). Response of Laminated Composite Flat Panels to Sonic Boom and Explosive Blast Loadings, AIAA J., 28(2): 345-352.

[17] Lam, K. and Chun, L. (1994). Analysis of Clamped Laminated Plates Subjected to Conventional Blast, Compos. Struct., 29(3): 311-321.

[18] Davison, L. (2008). Fundamentals of Shock Wave Propagation in Solids, Springer-Verlag, Berlin. 
[19] Lukyanov, A.A. (2010). An Equation of State of A Carbon-Fibre Epoxy Composite under Shock Loading, Eur. Phys. J. B, 74: 35-45.

[20] Cosserat, E. and Cosserat, F. (1909). Theorie des Corps Deformables, Hermann \& Fils, Paris, France.

[21] Mindlin, R.D. (1964). Micro-Structure in Linear Elasticity, Arch. Ration. Mech. Anal., 16: $51-78$.

[22] Eringen, C. and Suhubi, E.S. (1964). Nonlinear Theory of Micro-Elastic Solids II, Int. J. Eng. Sci., 2: 189-203.

[23] Bennett, T., Gitman, I.M. and Askes, H. (2007). Elasticity Theories with Higher-Order Gradients of Inertia and Stiffness for the Modelling of Wave Dispersion in Laminates, Int. J. Fract., 148: 185-193.

[24] Chen, W. and Fish, J. (2001). A Dispersive Model for Wave Propagation in Periodic Heterogeneous Media Based on Homogenization with Multiple Spatial and Temporal Scales, ASME J. Appl. Mech., 68: 153-161.

[25] Santosa, F. and Symes, W.W. (1991). A Dispersive Effective Medium for Wave Propagation in Periodic Composites, SIAM J. Appl. Math., 51: 984-1005.

[26] Wang, Z.P. and Sun, C.T. (2002). Modeling Micro-Inertia in Heterogeneous Materials under Dynamic Loading, Wave Motion, 36: 473-485.

[27] Engelbrecht, J., Berezovski, A., Pastrone, F. and Braun, M. (2005). Waves in Microstructured Materials and Dispersion, Philos. Mag., 85: 4127-4141.

[28] Crouch, R. and Oskay, C. (2010). Symmetric Meso-Mechanical Model for Failure Analysis of Heterogeneous Materials, Int. J. Mult. Comp. Eng., 8: 447-461.

[29] Oskay, C. and Fish, J. (2007). Eigendeformation-Based Reduced Order Homogenization for Failure Analysis of Heterogeneous Materials, Comput. Meth. Appl. Mech. Eng., 196(7): $1216-1243$.

[30] Yan, H., Oskay, C., Krishnan, A. and Xu, L. (2010). Compression after Impact Response of Woven Fiber-Reinforced Composites, Compos. Sci. Technol., 70: 2128-2136.

[31] Tekalur, S., Shivakumar, K. and Shukla, A. (2008). Mechanical Behavior and Damage Evolution 
in E-Glass Vinyl Ester and Carbon Composites Subjected to Static and Blast Loads, Composites Part B, 39(1): 57-65.

[32] Abu, A., Rashid, K. and Voyiadjis, G. (2006). A Finite Strain Plastic-Damage Model for High Velocity Impact Using Combined Viscosity and Gradient Localization Limiters: Part I-Theoretical Formulation, Int. J. Damage Mech., 15(4): 293-334.

[33] Voyiadjis, G., Abu, A. and Rashid, K. (2006). A Finite Strain Plastic-Damage Model for High Velocity Impacts Using Combined Viscosity and Gradient Localization Limiters: Part II-Numerical Aspects and Simulations, Int. J. Damage Mech., 15(4): 335-373.

[34] Perzyna, P. (1966). Fundamental Problems in Viscoplasticity, Adv. Appl. Mech., 9(2): 244-368.

[35] Simo, J.C. and Ju, J.W. (1987). Strain- and Stress-Based Continuum Damage Models - I. Formulation, Int. J. Solids Structures, 23(7): 821-840.

[36] Dube, J.F., Pijaudier-Cabot, G. and La Borderie, C. (1996). Rate Dependent Damage Model for Concrete in Dynamics, J. Engng. Mech., 122(10): 939-947.

[37] Needleman, A. (1988). Material Rate Dependence and Mesh Sensitivity in Localization Problems, Comput. Meth. Appl. Mech. Engng., 67: 69-86.

[38] Oskay, C. and Pal, G. (2010). A Multiscale Failure Model for Analysis of Thin Heterogeneous Plates, Int. J. Damage Mechanics, 19: 575-611.

[39] Oguni, K. and Ravichandran, G. (2001). Dynamic Compressive Behavior of Unidirectional E-glass/Vinylester Composites, J. Mater. Sci., 36(4): 831-838.

[40] Committee, A.I.H. (1992). ASM Handbook, ASM International.

[41] Roy, R., Sarkar, B. and Bose, N. (2001). Impact Fatigue of Glass Fibre-Vinylester Resin Composites, Composites Part A, 32(6): 871-876.

[42] Ochola, R., Marcus, K., Nurick, G. and Franz, T. (2004). Mechanical Behaviour of Glass and Carbon Fibre Reinforced Composites at Varying Strain Rates, Compos. Struct., 63(3-4): $455-467$. 


\section{FIGURE CAPTIONS}

Figure 1: Setup of EVE and Polyurea layered EVE panels: (a) front view of EVE composite panel, (b) side view of EVE composite panel, (c) representative finite element model of polyurea coated EVE composite panel, (d) E|P-conuration model, (e) P|E-configuration model, (f) $\mathrm{E}|\mathrm{P}| \mathrm{E}$-configuration model.

Figure 2: Blast loading profiles.

Figure 3: (a) The representative volume for the woven composite material, (b) reduced order model for the RVE with three constituents: fill (horizontal) and warp (vertical) fibers and the matrix (transparent).

Figure 4: Simulated stress-strain curves of EVE composite for quasi-static and high strain loading in compression and tension directions.

Figure 5: Comparison of experimental and numerically predicted center-point displacements of E-configuration panels subjected to input pressure of (a) $0.62 \mathrm{MPa}$, and, (b) $0.45 \mathrm{MPa}$.

Figure 6: Comparison of experimental and numerically predicted normalized center-point displacements of (a) $\mathrm{P} \mid$ E-configuration, (b) $\mathrm{E} \mid \mathrm{P}$-configuration, and, (c) $\mathrm{E}|\mathrm{P}| \mathrm{E}$-configuration panels subjected to input pressure of $0.75 \mathrm{MPa}$.

Figure 7: Displacement profiles of (a) experimentally observed E-panel when $P_{\text {inc }}=0.62 \mathrm{MPa}$;

(b) experimentally observed E|P-panel when $P_{\text {inc }}=0.75 \mathrm{MPa}$; [15] (c) simulated E-panel when $P_{\text {inc }}=0.62 \mathrm{MPa} ;$ (d) simulated E $\mid$ P-panel when $P_{\text {inc }}=0.75 \mathrm{MPa}$.

Figure 8: Effect of confinement on the evolution of center-point displacement in $\mathrm{E}|\mathrm{P}| \mathrm{E}$-configuration.

Figure 9: Effect of polyurea thickness on the evolution of center-point displacement in (a) $\mathrm{P} \mid$ E-configuration, (b) $\mathrm{E} \mid \mathrm{P}$-configuration, and, (c) $\mathrm{E}|\mathrm{P}|$ E-configuration. 


\section{TABLES}

Table 1: Calibrated parameters of the polyurea constitutive model (from Ref. [8]).

\begin{tabular}{ccccc}
\hline$\rho^{(p)}\left[\mathrm{kg} / \mathrm{m}^{3}\right]$ & $A^{(p)}$ & $B^{(p)}[\mathrm{K}]$ & $m^{(p)}[\mathrm{GPa} / \mathrm{K}]$ & $K_{\mathrm{ref}}^{(p)}[\mathrm{GPa}]$ \\
1070 & -10 & 107.54 & -0.015 & 4.948 \\
\hline$C_{t p}^{(p)}[\mathrm{K} / \mathrm{GPa}]$ & $T_{r e f}^{(p)}[\mathrm{K}]$ & $C_{V}^{(p)}\left[\mathrm{J} / \mathrm{mm}^{3} \mathrm{~K}\right]$ & $G_{\infty}^{(p)}[\mathrm{GPa}]$ & \\
7.2 & 273 & $1.977 \times 10^{-3}$ & 0.0224 \\
\hline$p_{1}^{(p)}$ & $p_{2}^{(p)}$ & $p_{3}^{(p)}$ & $p_{4}^{(p)}$ \\
0.8458 & 1.686 & 3.594 & 4.342 \\
\hline$q_{1}^{(p)}[\mathrm{ms}]$ & $q_{2}^{(p)}[\mathrm{ms}]$ & $q_{3}^{(p)}[\mathrm{ms}]$ & $q_{4}^{(p)}[\mathrm{ms}]$ \\
463.4 & 0.06407 & $1.163 \times 10^{-4}$ & $7.321 \times 10^{-7}$ & \\
\hline
\end{tabular}


Table 2: Properties and calibrated parameters of EVE composite.

\begin{tabular}{ccccccc}
\hline & $E[\mathrm{GPa}]$ & $K[\mathrm{GPa}]$ & $\rho\left[\mathrm{kg} / \mathrm{m}^{3}\right]$ & $\alpha\left[1 /{ }^{\circ} \mathrm{C}\right]$ & $C_{p}[\mathrm{~J} / \mathrm{gK}]$ & $T_{\text {ref }}[\mathrm{K}]$ \\
\cline { 2 - 6 } fiber & 70 & 33.3 & 2620 & $5.4 \times 10^{-6}$ & 0.810 & 298 \\
matrix & 3.4 & 1.62 & 1230 & $45 \times 10^{-6}$ & 1.05 & 298 \\
\hline & $a$ & $b$ & $c_{1}$ & $q$ & $p$ & \\
\cline { 2 - 6 } warp & 0.17 & 6 & -22 & $10^{-8}$ & 2 & \\
fill & 0.32 & 6 & 0 & $10^{-8}$ & 2 & \\
matrix & 1.4 & 2 & 0 & $2 \times 10^{-6}$ & 2 & \\
\hline
\end{tabular}


Table 3: EVE composite properties under quasi-static loading.

\begin{tabular}{lllllll}
\hline & & \multicolumn{2}{c}{ Modulus [GPa] } & \multicolumn{2}{c}{ Strength [MPa] } & \multirow{2}{*}{ Poisson's ratio } \\
& & Tension & Compression & Tension & Compression & \\
\hline \multirow{2}{*}{ warp } & Experiment [31] & 29.2 & 31.9 & 512.5 & 363.4 & 0.16 \\
& Simulation & 29.0 & 29.0 & $516.7^{(0.82 \%)^{1}}$ & $369.7^{(1.7 \%)}$ & 0.15 \\
\multirow{2}{*}{ fill } & Experiment [31] & 23.9 & 26.9 & $350.9^{(3.6 \%)}$ & 336.4 & 0.14 \\
& Simulation & 24.5 & 24.5 & $363.6^{(0.45 \%)}$ & 0.15 \\
\hline
\end{tabular}

${ }^{1}$ difference between simulated and experimental results 


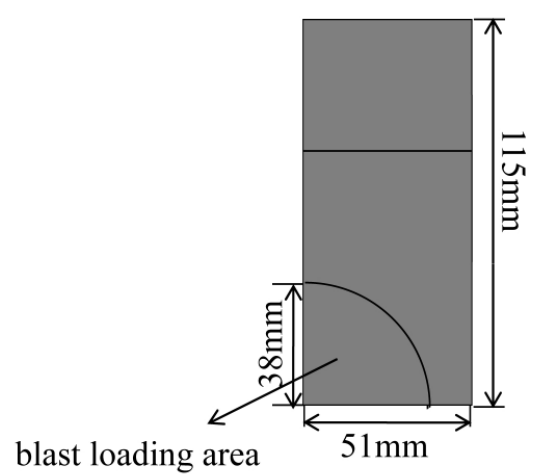

(a)

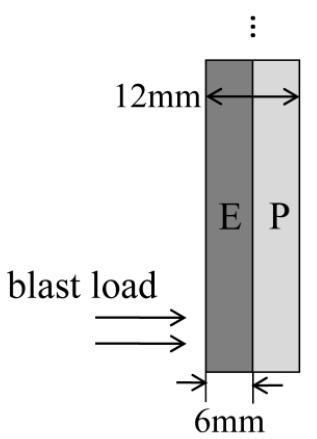

(d)

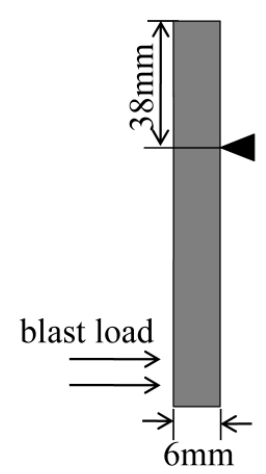

(b)

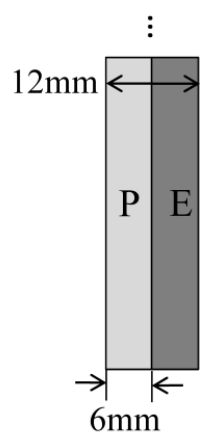

(e)

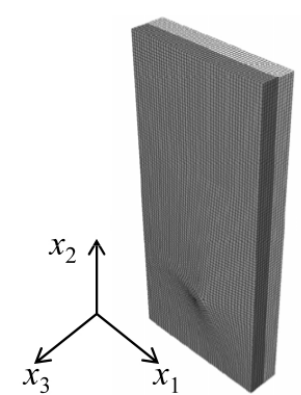

(c)

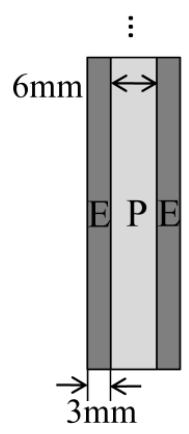

(f)

Figure 1



Time

Figure 2 


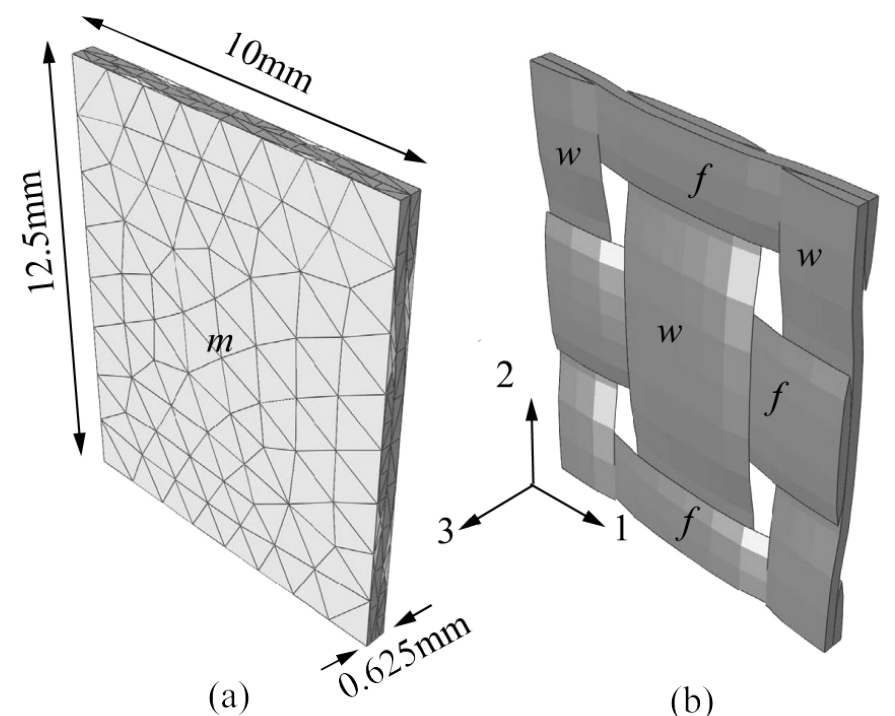

Figure 3

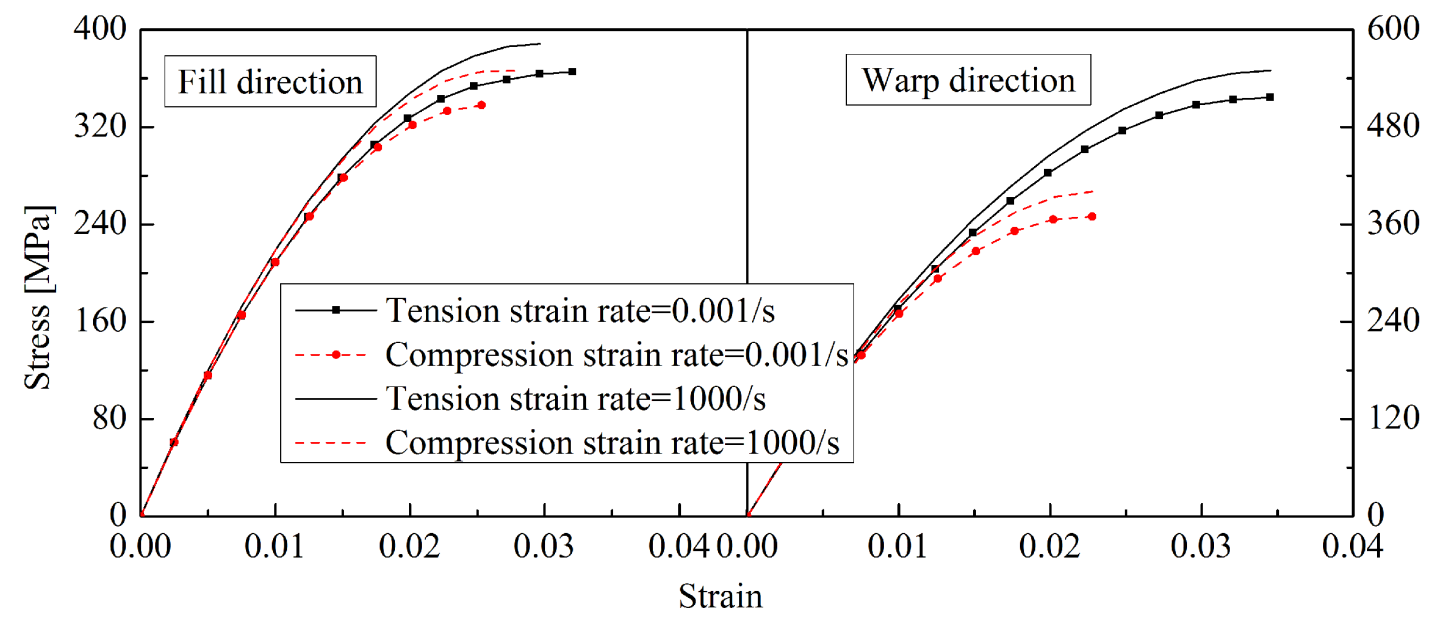

Figure 4 


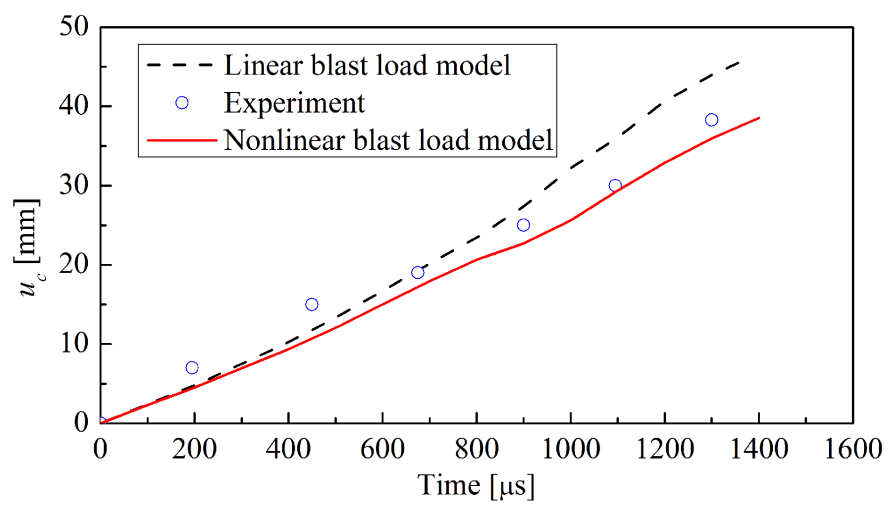

(a)

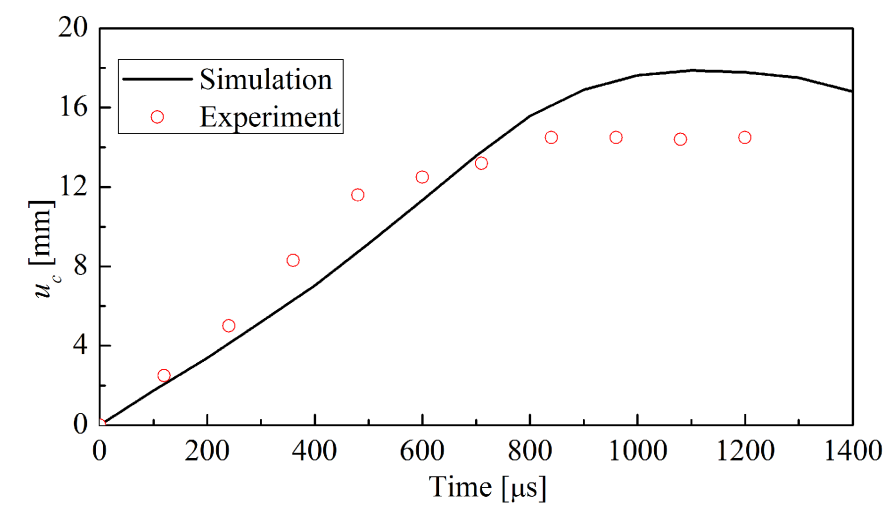

(b)

Figure 5 




(a)

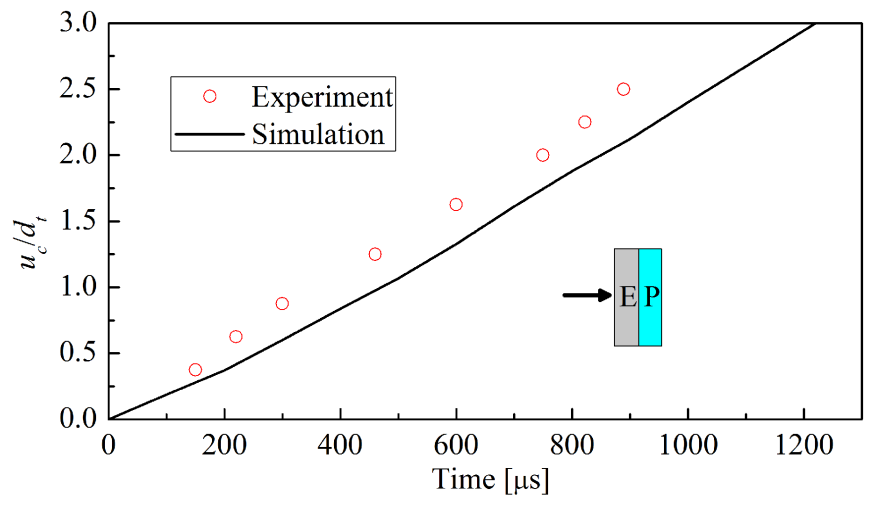

(b)

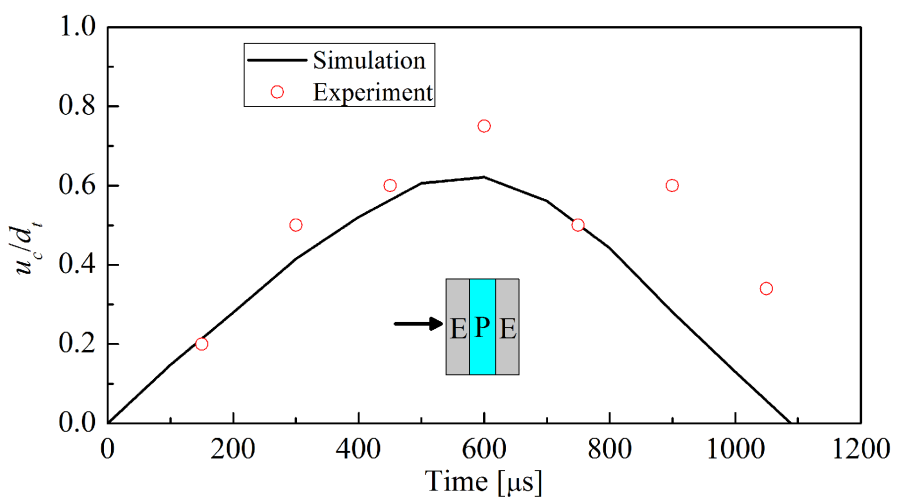

(c)

Figure 6 


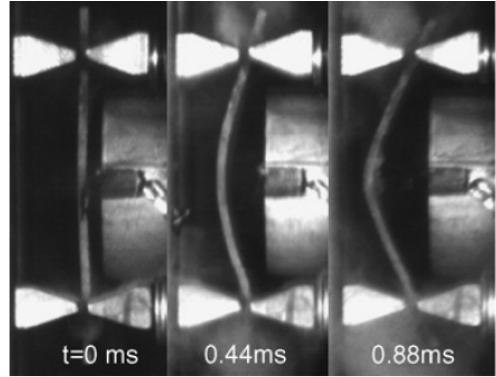

$\omega^{(\mathrm{m})}$

$+1.000 \mathrm{e}+00$

$+9.167 \mathrm{e}-01$

$+8.333 \mathrm{e}-0$

$+7.500 \mathrm{e}-01$

$+6.667 \mathrm{e}-0$

$+5.833 \mathrm{e}-0$

$+5.000 \mathrm{e}-0$

$+4.167 \mathrm{e}-01$

$+3.333 \mathrm{e}-01$

$+2.500 \mathrm{e}-0$

$+1.667 \mathrm{e}-01$

$+8.333 \mathrm{e}-02$

$+0.000 \mathrm{e}+00$

$$
\mathrm{t}=0 \mathrm{~ms}
$$

$0.4 \mathrm{~ms}$

(c)

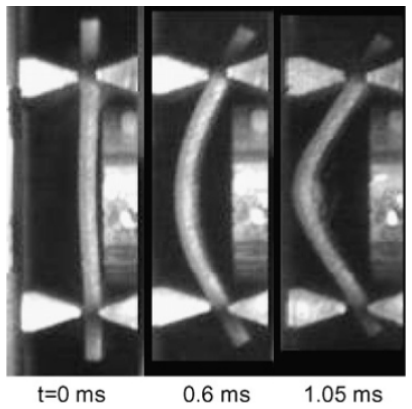

(b)


(d)

Figure 7

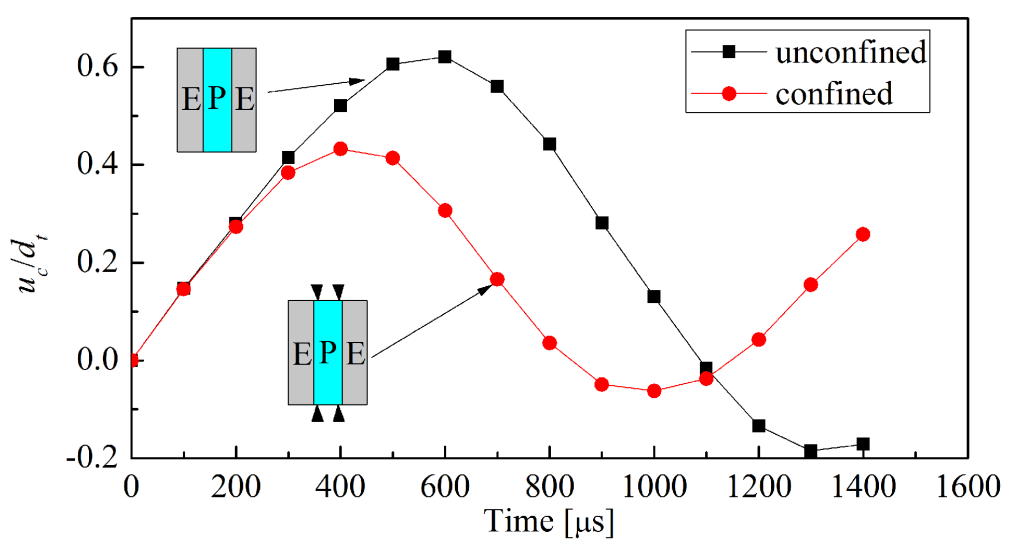

Figure 8 


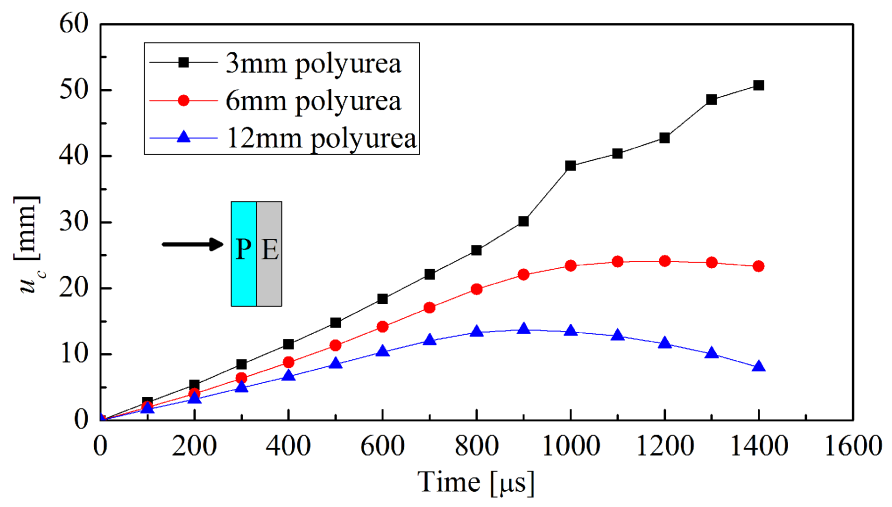

(a)

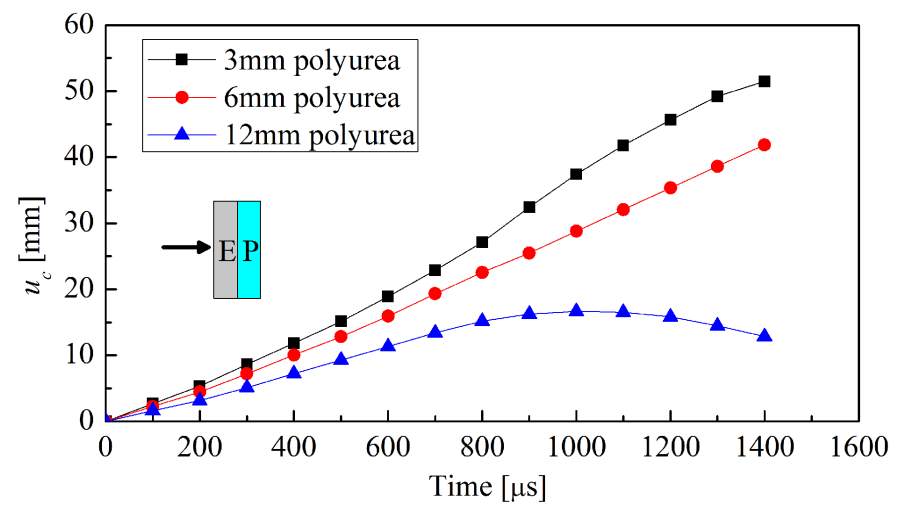

(b)

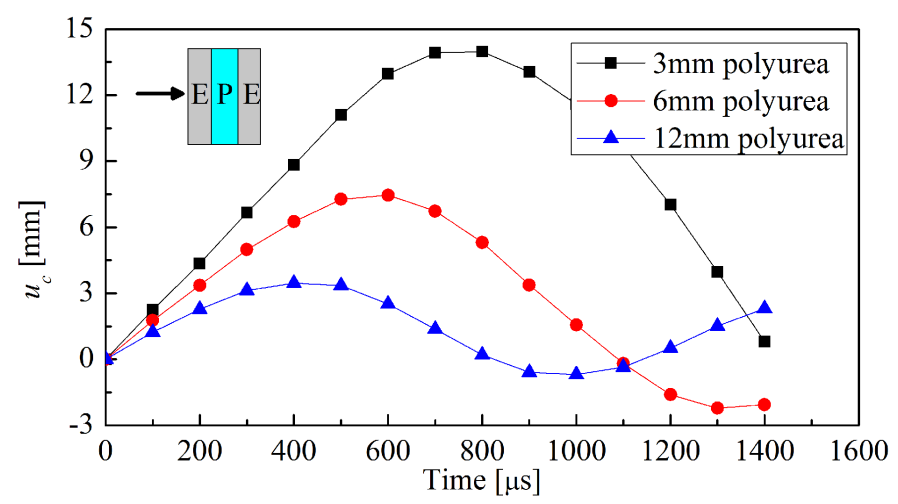

(c)

Figure 9 